\title{
Mapping the rotational diffusion of fluorophores in cells with time-resolved wide-field fluorescence anisotropy imaging
}

\author{
K. Suhling ${ }^{1,2,3}$, J. Siegel ${ }^{1,5}$, S. Lévêque-Fort ${ }^{1,6}$, S.E.D. Webb $^{1}$, P. Lanigan $^{1}$, Y. Sabharwal ${ }^{4}$, D. Phillips ${ }^{3}$, D.M. Davis ${ }^{2}$ \\ and P.M.W. French ${ }^{l}$ \\ ${ }^{1}$ Photonics Group, Department of Physics, ${ }^{2}$ Department of Biological Sciences, ${ }^{3}$ Department of Chemistry, Imperial \\ College of Science, Technology \& Medicine, London SW7 2BW, UK, tel +44 207594 7715, fax +44 2075947714 \\ ${ }^{4}$ Optical Insights, LLC, $18072^{\text {nd }}$ Street, Suite 1, Santa Fe, NM 87505, USA \\ 5 present address: Instituto de Optica (CSIC), 28006 Madrid, Spain \\ ${ }^{6}$ present address: Laboratoire de Photophysique Moleculaire, 91405 Orsay, France
}

Abstract: We demonstrate time-resolved fluorescence anisotropy imaging with simultaneous acquisition of the parallel and perpendicular polarization of a fluorescence image. This allows imaging of the viscosity in cells.

\section{Summary}

For analysis of sample arrays in assays and for imaging of molecular biology, it is desirable to develop techniques for contrasting both different biological species and also the local environment of such species. Fluorescent labels provide a means not only to visualise the distribution of target species but also to probe their local environment through the fluorescence lifetime. This approach is attractive since it is based on relative intensity measurements and is independent of fluorophore concentration. Here, we report the extension of picosecond time-gated fluorescence lifetime imaging (FLIM) to time-resolved fluorescence anisotropy imaging (TR-FAIM). ${ }^{1}$ Upon excitation with linearly polarized laser pulses, the parallel and perpendicular components of the fluorescence emission are imaged simultaneously using a Polarization-Resolved Imager. This yields both the rotational correlation time and fluorescence lifetime in each pixel of the image, the former providing information about the rotational diffusion of a fluorophore. To demonstrate the technique, we imaged $B$ cells using the fluorescein derivative CFSE (carboxyfluorescein diacetate succinimidyl ester), which stains the cytoplasm. The FLIM image shows a homogeneous distribution of the fluorophore and has an average fluorescence lifetime of $3.46 \pm 0.16 \mathrm{~ns}$ (Fig 1a)). The rotational correlation time reports on the viscosity of the cytoplasm, and the corresponding preliminary image is shown in Fig 1b). The average value for the rotational correlation time is $4.50 \pm 0.87 \mathrm{~ns}$ at $20^{\circ} \mathrm{C}$, which corresponds to an average cytoplasmic viscosity of $14 \mathrm{cp}$. This is in good agreement with $10-13 \mathrm{cp}$ at $23^{\circ} \mathrm{C}$ quoted previously. ${ }^{2}$ This illustrates the potential to image, for example, viscosity variations in live cells. Moreover, as the rotational diffusion can be slowed down by binding as well as other conformational changes, the technique has potential to visualise, for example, the binding of ligands and receptors

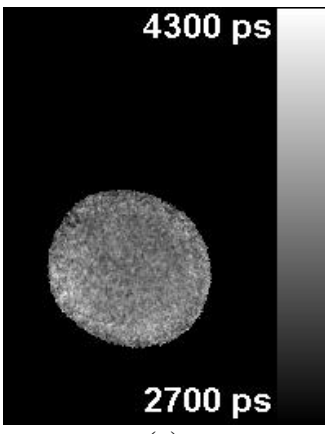

(a)

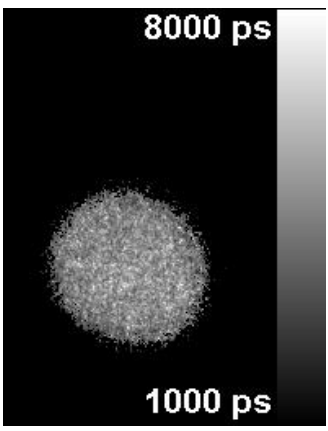

(b)

Fig 1: a) A FLIM image of a B cell stained with the fluorescein derivative CFSE. b) The corresponding rotational correlation time image. The images where acquired simultaneously in 75 seconds.

\section{References}

[1] Wide-field time-resolved fluorescence anisotropy imaging (TR-FAIM) - Imaging the rotational mobility of a fluorophore. J. Siegel, K. Suhling, S. Lévêque-Fort, S.E.D. Webb, D.M. Davis, D. Phillips, Y. Sabharwal and P.M.W. French. Review of Scientific Instruments, in press (Jan 2003).

[2] Mapping of fluorescence anisotropy in living cells by ratio imaging. Applications to cytoplasmic viscosity. J.A. Dix and A.S. Verkman. Biophysical Journal, 57, 231-240, 1990. 